\title{
BIOMONITOREO DE METALES PESADOS EN PLUMAS DE AVES ACUÁTICAS RESIDENTES DEL LAGO DE CHAPALA, MÉXICO
}

\author{
Deisy GONZÁLEZ1 ${ }^{1}$, Dioselina ÁLVAREZ BERNAL ${ }^{1 *}$, Miguel MORA ${ }^{2}$, \\ Héctor René BUELNA-OSBEN ${ }^{1}$ y Jorge Ricardo RUELAS-INSUNZA ${ }^{3}$
}

${ }^{1}$ Centro Interdisciplinario de Investigación para el Desarrollo Integral Regional, Unidad Michoacán, Instituto Politécnico Nacional, Justo Sierra 28, Col. Centro, 59510 Jiquilpan, Michoacán, México

${ }^{2}$ Department of Wildlife and Fisheries Sciences, Texas A\&M University, 454 Throckmorton St, College Station, TX 77840, EUA

${ }^{3}$ Instituto Tecnológico de Mazatlán, Sección Ambiental, apartado postal 757, 82000 Mazatlán, Sinaloa

*Autora para correspondencia: dalvarezb@ipn.mx

(Recibido febrero 2017; aceptado septiembre 2017)

Palabras clave: Ardea alba, contaminación, humedal, Egretta thula, Nycticorax nycticorax, sitio RAMSAR

\section{RESUMEN}

El lago de Chapala es considerado importante por los servicios económicos, recreativos y ecológicos que ofrece. Es abastecido principalmente por el río Lerma, en el cual se vierten los desechos industriales de los estados de México, Querétaro y Guanajuato, así como los desechos urbanos y agropecuarios de los estados de Michoacán y Jalisco. Estos desechos pueden ser nocivos para los organismos residentes en el lago de Chapala, ya que algunos compuestos químicos tienen la capacidad de acumularse a través de la red trófica y causar daños a dichas poblaciones. En este trabajo se determinó la contaminación por metales pesados mediante la cuantificación de Ag, As, Cd, $\mathrm{Cr}, \mathrm{Hg}, \mathrm{Pb}, \mathrm{Sr}$ y Ti en plumas de tres especies locales de aves acuáticas (Ardea alba, Egretta thula y Nycticorax nycticorax), así como en el sedimento y el agua del lago de Chapala. El análisis de la concentración de metales en plumas se realizó mediante espectrometría con fuente de plasma de acoplamiento inductivo. Ardea alba presentó la mayor concentración promedio de $\mathrm{Pb}(43.65 \mu \mathrm{g} / \mathrm{g})$ y $\mathrm{Cr}(52.47 \mu \mathrm{g} / \mathrm{g})$ en el raquis de las plumas. Eestas concentraciones fueron estadísticamente significativas respecto a las encontradas en E. thula y $N$. nycticorax $(\mathrm{p} \leq 0.05)$. Los valores obtenidos del factor de acumulación biota-sedimento indican acumulación de $\mathrm{Ag}, \mathrm{As}, \mathrm{Cd}, \mathrm{Cr}, \mathrm{Cu}$, $\mathrm{Hg}$ y $\mathrm{Pb}$ en las aves del lago de Chapala. Las plumas de aves acuáticas residentes en dicho lago demostraron ser una herramienta no letal y útil para el biomonitoreo de la contaminación por metales pesados.

Key words: Ardea alba, pollution, wetland, Egretta thula, Nycticorax nycticorax, RAMSAR site

\begin{abstract}
Lake Chapala is considered important because of the economic, recreational, and ecological services that it offers. The main water source of the lake is the Lerma river, which receives industrial waste discharges from the states of Mexico, Queretaro, and Guanajuato, as well as municipal and agricultural waste discharges from the states of Michoacan and Jalisco. Contaminants present in such waste discharges can accumulate through the food web and affect other species in the Chapala ecosystem. We estimated
\end{abstract}


heavy metal pollution in the lake by measuring concentrations of $\mathrm{Ag}, \mathrm{As}, \mathrm{Cd}, \mathrm{Cr}, \mathrm{Hg}$, $\mathrm{Pb}, \mathrm{Sr}$ and $\mathrm{Ti}$ in water and sediments, and in feathers from three resident aquatic birds (Ardea alba, Egretta thula, Nycticorax nycticorax). The analysis of heavy metals in feathers was performed using inductively-coupled mass spectrometry. A. alba had significantly greater concentrations of $\mathrm{Pb}(43.65 \mu \mathrm{g} / \mathrm{g})$ and $\mathrm{Cr}(52.47 \mu \mathrm{g} / \mathrm{g})$ in feathers (rachis) than E. thula and $N$. nycticorax $(\mathrm{p} \leq 0.05)$. The biota-sediment bioaccumulation factors suggest accumulation of $\mathrm{Ag}, \mathrm{As}, \mathrm{Cd}, \mathrm{Cr}, \mathrm{Cu}, \mathrm{Hg}$ and $\mathrm{Pb}$ in birds from Lake Chapala. Feathers from resident aquatic bird species in Lake Chapala can be used as a non-lethal procedure useful for monitoring heavy metal contamination.

\section{INTRODUCCIÓN}

La bioacumulación de metales se presenta cuando las concentraciones de un contaminante son mayores en el organismo que en el medio que lo rodea (Beek et al. 2000). Usualmente se utilizan organismos como indicadores de bioacumulación, debido a que reflejan la distribución de los contaminantes en los diferentes niveles de las cadenas tróficas. Los organismos más adecuados para ser considerados como bioindicadores deben ser residentes, es decir, que se encuentren en su hábitat natural, y además debe haber abundancia de dicha especie. Por otro lado, su muestreo debe ser fácil, lo cual se relaciona con el tamaño y la representatividad de su especie; también es importante que tengan la capacidad para concentrar los contaminantes sin que ello les provoque la muerte (Parra 2014). Usar aves como bioindicadoras de contaminación ambiental tiene ciertas ventajas debido a que se encuentran en los distintos eslabones de las cadenas tróficas. Esto permite evaluar las distintas rutas de exposición y acumulación de contaminantes a través de éstas, además de estar relacionado con la salud ambiental y humana, debido a que las aves acuáticas son piscívoras y comparten el alimento con los seres humanos (Estrada-Guerrero y Soler-Tovar 2014). Existen varios estudios en los que se utilizan aves para el biomonitoreo de la contaminación ambiental (Honda et al. 1986, Custer et al. 2008, Ferreira 2011). Otra ventaja es que muchas aves son residentes de los lugares que se pretende investigar, por lo que pueden proporcionar más información del sitio (Burger et al. 2008, Burger 2013). Algunas técnicas utilizadas en el biomonitoreo de contaminantes son invasivas y letales, y en ellas se utilizan distintas partes de las aves como el hígado y los riñones, ya que muchos de los metales son lipofílicos y tienden a acumularse en esos órganos. También se utilizan las vísceras, sangre, músculo y huesos (Burger y Gochfeld 2004, Nam et al. 2005).
Existen otros métodos no letales y no invasivos en los que no se perturba a la población de aves con la que se va a trabajar. Estas técnicas toman como unidad de muestra las plumas y cascarones de huevo o huevos completos (Zhang y Ma 2011, Skoric et al. 2012).

Las plumas se han utilizado como unidades de monitoreo de contaminantes debido a que los metales pesados que circulan por el torrente sanguíneo se adhieren a las proteínas de las plumas (Dauwe et al. 2003), por lo que se acumulan durante el desarrollo y crecimiento de las mismas (Lodenius y Solonen 2013). Una vez dentro de la pluma, los metales pueden ligarse con moléculas de proteínas y ser inmovilizados; cuando la pluma deja de crecer es sellada y deja de tener interacción con la fisiología del ave, lo cual le permite reflejar los metales acumulados durante la época de crecimiento o muda (Dauwe et al. 2003, Nam et al. 2005, Burger et al. 2008). El uso de plumas como bioindicadores ofrece ciertas ventajas, entre ellas su fácil recolección y almacenamiento. Sus desventajas son que los niveles varían de acuerdo con el periodo de muda, el tipo de pluma y la contaminación externa (García-Fernández et al. 2013).

El lago de Chapala tiene importancia económica debido a que abastece de agua potable a la ciudad de Guadalajara, y en él se realizan actividades como la pesca, que es la principal fuente de ingresos de un gran número de la familias que viven cerca de este cuerpo de agua (Rosales-Hoz et al. 2000). Por otro lado, tiene importancia ecológica y en 2011 fue catalogado como sitio RAMSAR por ser considerado un humedal de gran importancia donde se puede encontrar una gran diversidad de especies. Cuenta con 29 especies, incluidas nativas y exóticas (Moncayo-Estrada y Buelna-Osben 2001). También se encuentra una gran diversidad de aves acuáticas (aproximadamente 80 especies) (Torres et al. 2014). En promedio se mantiene una población aproximada de más de 20000 aves, principalmente en invierno, cuando se puede destacar la presencia del pelícano 
blanco (Pelecanus erythrorhynchos Gmelin 1789), que es una especie migratoria y algunas residentes como la garza blanca (Ardea alba Linnaeus 1758), garza de pie dorado (Egretta thula Molina 1782) y el perrito de agua (Nycticorax nycticorax Linnaeus 1758) (Gutiérrez et al. 2008).

El lago de Chapala es alimentado por el río Lerma, cuya parte alta recibe aguas residuales de origen químico, metalúrgico y textil, entre otras industrias (Brito et al. 2015). La parte media recibe aguas derivadas de desechos de plantas termoeléctricas, petroquímicas, de fertilizantes, empacadoras de frutas, granjas avícolas y porcícolas e industrias curtidoras, al igual que aguas negras no tratadas y desechos de las zonas agrícolas (Sedeño-Díaz y López-López 2007). Dichas aguas son portadoras de metales pesados como $\mathrm{Cu}, \mathrm{Cd}, \mathrm{Pb}, \mathrm{Hg}, \mathrm{As}$ y $\mathrm{Cr}^{+6}$, entre otros (Estrada-Guerrero y Soler-Tovar 2014, Parra 2014). Los metales pesados asociados con estas descargas frecuentemente se acumulan en organismos acuáticos y pueden causarles daños individuales y poblacionales. Las aves acuáticas que se eligieron en este estudio fueron A. alba, E. thula y $N$. nyticorax. Estas especies anidan y permanecen en la ribera del lago, por lo tanto, se consideran residentes anuales. Las tres especies seleccionadas se alimentan principalmente de peces y crustáceos, los cuales a su vez se alimentan de lo que crece o está en los sedimentos (Trujillo-Cárdenas et al. 2010). Las aves acuáticas se han utilizado para el biomonitoreo de metales pesados en otros lugares del mundo (Honda et al. 1986, Custer et al. 2008, Ferreira 2011).

No hay reportes del uso de aves residentes del lago de Chapala como bioindicadores de contaminación por metales pesados. Por lo tanto, en este trabajo se determinó la contaminación por metales pesados y el factor de bioacumulación en plumas de $A$. alba, E. thula y N. nyticorax mediante el análisis de Ag, As, $\mathrm{Cd}, \mathrm{Cr}, \mathrm{Hg}, \mathrm{Pb}, \mathrm{Sr}$ y Ti.

\section{MATERIALES Y MÉTODOS}

\section{Área de estudio}

El lago de Chapala se encuentra en la meseta central de México, entre los estados de Jalisco y Michoacán, y es el lago tropical más grande del país. Este estudio se llevó a cabo en la orilla del lago de Chapala que colinda con la región de Petatán, Michoacán, México (20 $07^{\circ} \mathrm{N}, 102^{\circ} 50^{\prime}$ O) (Fig. 1), a una altura de $1520 \mathrm{msnm}$. Su clima es templado con lluvias en verano, precipitación pluvial anual de
$800 \mathrm{~mm}$, y temperaturas que varían de 10.4 a $25.4{ }^{\circ} \mathrm{C}$ (INEGI 2016).

\section{Recolección de muestras}

La recolección de las muestras se realizó completamente al azar en la época de reproducción de las aves (de junio a agosto de 2014) en la orilla del lago, específicamente en la zona de alimentación y anidación, con la finalidad de garantizar que las plumas fueran de las aves seleccionadas para el estudio. En este periodo se recolectaron alrededor de 20 plumas para cada especie adulta. Las muestras de plumas se colocaron en bolsas plásticas con cierre hermético, y se etiquetaron para su posterior análisis. Las muestras de sedimento y agua se colectaron en 27 puntos alrededor del lago, aproximadamente cada $5 \mathrm{~km}$ entre una y otra estación de muestreo con la ayuda de un sistema geoposicionador (GPS, por sus siglas en inglés) tipo eTrex 30, Garmin ${ }^{\circledR}$, de acuerdo con la metodología de Torres et al. (2016). Los parámetros característicos del agua del lago se determinaron in situ con una sonda multiparamétrica Hydrolab ${ }^{\circledR}$ modelo DS5X (OTT Hydromet, Loveland, EUA) y se confirmaron en laboratorio; en promedio fueron los siguientes: $\mathrm{pH} 8.8$, conductividad eléctrica $1.1 \mathrm{dS} / \mathrm{m}$, temperatura $20.4{ }^{\circ} \mathrm{C}, 96.8 \%$ de oxígeno disuelto, turbidez 39.2 unidades nefelométricas de turbidez (ntu, por sus siglas en inglés).

\section{Preparación y análisis de muestras}

Las plumas recolectadas se lavaron tres veces con acetona y posteriormente con agua destilada. Se dejaron secar al aire durante la noche a temperatura ambiente para eliminar el exceso de humedad. Ya secas, se cortaron en fragmentos pequeños, separando la barba y el raquis de la pluma, y se pulverizaron en un mortero de porcelana (Burger et al. 2008, Misztal-Szkudlinska et al. 2012). La digestión ácida de las muestras de plumas (raquis y barba), agua y sedimentos se realizó según la técnica propuesta por Ruelas-Insunza et al. (2009). La determinación de las concentraciones de metales pesados (Ag, $\mathrm{Al}$, $\mathrm{As}, \mathrm{Cr}, \mathrm{Cd}, \mathrm{Cu}, \mathrm{Pb}$ y $\mathrm{Ti}$ ) en las muestras digeridas se realizó mediante un ICP (Perkin Elmer Optimal Emission Spectrometer Optima 4300 DV). Para la detección de $\mathrm{Hg}$, las muestras digeridas se colocaron en viales, se diluyeron con $15 \mathrm{ml}$ de agua Milli-Q para hacer submuestras por triplicado y determinar este elemento después de la reducción con $\mathrm{SnCl}_{2}$ en un analizador de mercurio Buick Scientific modelo 410 (Ruelas-Insunza et al. 2011a), con un límite de detección de $0.01 \mu \mathrm{g} / \mathrm{g}$ y un porcentaje de recuperación de $99.6 \%$. 


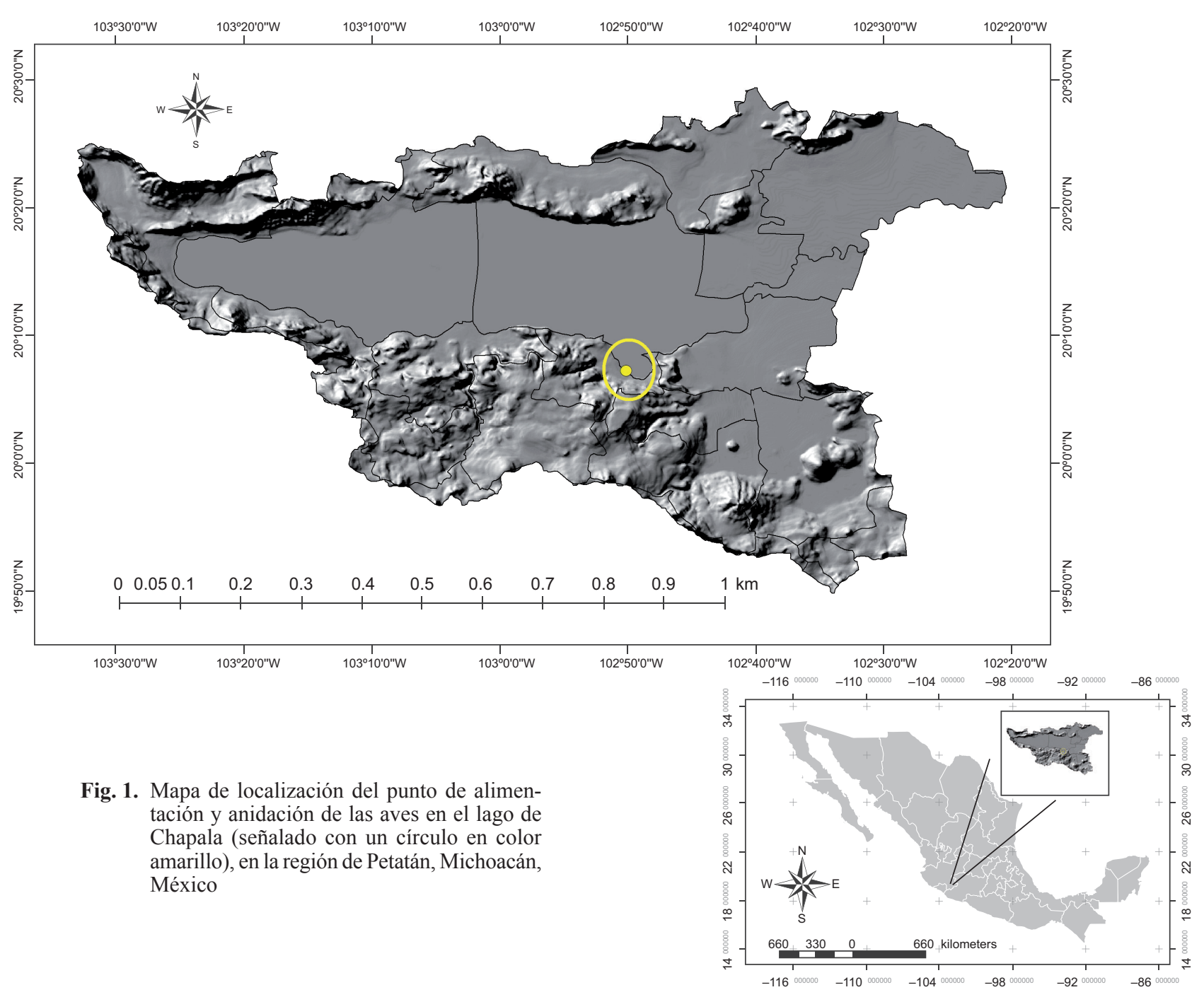

Factor de bioacumulación de sedimento a biota

Se utilizó el factor de bioacumulación de sedimento a biota (FBSB) para establecer la relación ecotoxicólogica entre las especies y su hábitat, debido a que los peces y crustáceos con los que se alimentan las aves crecen, se reproducen e incluso también se alimentan en el área de los sedimentos (Trujillo-Cárdenas et al. 2010). El FBSB se calculó con la siguiente fórmula:

$\mathrm{FBSB}=C_{\mathrm{B}} / C_{\mathrm{S}}$

donde $C_{\mathrm{B}}$ es la concentración del contaminante en el ave y $C_{\mathrm{S}}$ es la concentración del contaminante en el sedimento. Un FBSB $>1$ indica el potencial de bioacumulación del metal en relación con los niveles en el sedimento (Calle et al. 2015).

\section{Análisis estadístico}

Para determinar si las concentraciones medias de los metales pesados son diferentes entre especies de aves se utilizó un análisis de varianza de un factor. Se estableció un valor de probabilidad de $5 \%(\mathrm{p}<$ $0.05)$ y se implementó la prueba de comparación múltiple de Tukey para identificar cuáles medias de los valores obtenidos para las especies son significativamente diferentes. Se utilizó el paquete estadístico SAS $9.1^{\circledR}$ (SAS 2004).

\section{RESULTADOS Y DISCUSIÓN}

Las concentraciones de metales pesados (Ag, As, $\mathrm{Cr}, \mathrm{Cd}, \mathrm{Cu}, \mathrm{Hg}, \mathrm{Pb}$ y $\mathrm{Ti}$ ) obtenidos en las plumas de las aves estudiadas, así como en las muestras de 
agua y sedimento, se presentan en el Cuadro I. Las concentraciones de metales en el agua fueron bajas (0.01-0.03 $\mu \mathrm{g} / \mathrm{mL})$ en comparación con las concentraciones en los sedimentos $(0.04-118.8 \mu \mathrm{g} / \mathrm{g})$, principalmente para $\mathrm{Ti}(118.8 \mu \mathrm{g} / \mathrm{g})$. El pH del agua del lago de Chapala es básico (8.8), por lo que tiende a incrementar la toxicidad y afectar la solubilidad de los metales. Esta condición puede hacer que algunos metales se precipiten y depositen en los sedimentos (Förstner 1981). Las muestras de agua y sedimento se colectaron durante febrero y marzo de 2014. Las tendencias de los metales siguieren el siguiente patrón en el agua del lago: $\mathrm{Ti}>\mathrm{Pb}=\mathrm{Cr}=\mathrm{Cd}=\mathrm{As}>$ $\mathrm{Ag}=\mathrm{Cu}=\mathrm{Hg}$. De igual manera, las concentraciones en sedimentos variaron de la siguiente forma: $\mathrm{Ti}>$ $\mathrm{Cr}>\mathrm{Cu}>\mathrm{Pb}>\mathrm{Cd}>\mathrm{Hg}>\mathrm{Ag}=\mathrm{As}$, lo que significa que el metal que más se encuentra en el agua y en los sedimentos es el Ti.

Las concentraciones de metales en plumas de aves acuáticas variaron de acuerdo con el siguiente patrón: raquis de $A$. alba: $\mathrm{Cr}>\mathrm{Pb}>\mathrm{Cu}>\mathrm{Ti}>\mathrm{As}>$ $\mathrm{Cd}>\mathrm{Ag}>\mathrm{Hg}$; barba de A. alba y E. thula: $\mathrm{Cr}>\mathrm{Cu}$ $>\mathrm{Ti}>\mathrm{Pb}>\mathrm{As}>\mathrm{Cd}>\mathrm{Ag}>\mathrm{Hg}$; raquis de E. thula y barba de $N$. nycticorax: $\mathrm{Cr}>\mathrm{Ti}>\mathrm{Cu}>\mathrm{Pb}>\mathrm{As}$ $>\mathrm{Cd}>\mathrm{Ag}>\mathrm{Hg}$; raquis de N. nycticorax: $\mathrm{Cr}>\mathrm{Cu}$ $>\mathrm{Ti}>\mathrm{As}>\mathrm{Pb}>\mathrm{Cd}>\mathrm{Ag}>\mathrm{Hg}$. Lo anterior indica que cada especie concentra los metales en diferente orden, sin embargo, se aprecia que el $\mathrm{Cr}$ es el metal que mayormente se concentra y que $\mathrm{Ag}$ y $\mathrm{Hg}$ son los que se encuentra en menor concentración.

A continuación se describe la variación de cada uno de los elementos detectados en plumas.

\section{Cromo}

El elemento con mayor concentración en plumas de aves fue el $\mathrm{Cr}$ (Cuadro I); sin embargo, sólo se aprecia diferencia significativa entre la barba de A. alba y el raquis de $N$. nycticorax $(\mathrm{p}=0.0023)$. Al- gunos estudios han reportado para distintas colonias en una misma zona concentraciones de $1.03,0.88 \mathrm{y}$ $0.60 \mu \mathrm{g} / \mathrm{g}$ (Padula et al. 2010). Custer et al. (2008) encontraron concentraciones de $3.28,3.16$ y 2.48 $\mu \mathrm{g} / \mathrm{g}$ en $N$. nycticorax en tres distintas colonias de una misma zona; Abdullah et al. (2015) obtuvieron concentraciones de 21.1 y $19 \mu \mathrm{g} / \mathrm{g}$ de $\mathrm{Cr}$ en Bubulcus ibis en dos localidades de la misma ciudad. Las concentraciones encontradas en aves acuáticas del lago de Chapala son dos a cinco veces mayores que las encontradas en los estudios citados. Este metal se encuentra en aguas residuales de industrias de curtiduría, metalurgia, pinturas, cerámicas, textiles, fungicidas, ladrillos y pigmentos (Förstner 1981). Hay alrededor de 3500 industrias a lo largo de la cuenca Lerma-Chapala que vierten sus efluentes al río Lerma (Hansen y van Afferden 2004). Esta puede ser la razón por la que este metal sea el más abundante en las tres especies de aves residentes del lago de Chapala. El cromo hexavalente $\left(\mathrm{Cr}^{+6}\right)$ en aves es tóxico y puede causar trastornos endocrinos, reproductivos y malformaciones congénitas, además de ser un potente carcinógeno (Manjula et al. 2015, Sfakianakis et al. 2015).

\section{Titanio}

Se encontraron diferencias significativas en las concentraciones de $\mathrm{Ti}$ en la barba de las plumas de especies de garzas. E. thula tuvo la concentración más alta $(33.70 \mu \mathrm{g} / \mathrm{g})$, seguida por $N$. nycticorax con una concentración de $30.69 \mu \mathrm{g} / \mathrm{g}$, en tanto que $A$. alba presentó la concentración más baja $(11.04 \mu \mathrm{g} / \mathrm{g})(\mathrm{p}$ $=0.026$ ). En el raquis de la pluma no se observaron diferencias significativas entre especies, lo que puede deberse a que este metal no se encuentra en formas biodisponibles (Förstner 1981). El Ti es uno de los elementos más abundantes en la corteza terrestre y no se ha reportado que provoque daños en aves.

CUADRO I. VALORES PROMEDIO DE LOS METALES PESADOS EN AVES, AGUA Y SEDIMENTO DEL LAGO DE CHAPALA (CONCENTRACIONES REPORTADAS EN $\mu \mathrm{g} / \mathrm{g}$ Y EN $\mu \mathrm{g} /$ mL PARA EL AGUA DEL LAGO)

\begin{tabular}{lllllllll}
\hline Matriz & $\mathrm{Ag}$ & $\mathrm{As}$ & $\mathrm{Cd}$ & $\mathrm{Cr}$ & $\mathrm{Cu}$ & $\mathrm{Hg}$ & $\mathrm{Pb}$ & $\mathrm{Ti}$ \\
\hline Ardea alba raquis & $5.4^{\mathrm{a}}$ & $8.2^{\mathrm{a}, \mathrm{b}}$ & $12.6^{\mathrm{a}}$ & $29.8^{\mathrm{a}, \mathrm{b}}$ & $20.5^{\mathrm{b}, \mathrm{c}}$ & $0.36^{\mathrm{a}, \mathrm{b}}$ & $8.7^{\mathrm{a}}$ & $11.0^{\mathrm{b}, \mathrm{c}}$ \\
A. Alba barba & $4.2^{\mathrm{b}}$ & $5.9^{\mathrm{b}}$ & $5.7^{\mathrm{a}, \mathrm{b}}$ & $99.2^{\mathrm{a}}$ & $27.8^{\mathrm{a}, \mathrm{b}}$ & $0.62^{\mathrm{a}, \mathrm{b}}$ & $7.1^{\mathrm{a}}$ & $11.0^{\mathrm{b}, \mathrm{c}}$ \\
Egretta thula barba & $5.4^{\mathrm{a}}$ & $7.7^{\mathrm{a}, \mathrm{b}}$ & $7.2^{\mathrm{a}, \mathrm{b}}$ & $33.9^{\mathrm{a}, \mathrm{b}}$ & $34.6^{\mathrm{a}}$ & $1.1^{\mathrm{a}}$ & $8.9^{\mathrm{a}}$ & $33.7^{\mathrm{b}}$ \\
E. thula raquis & $5.4^{\mathrm{a}}$ & $9.4^{\mathrm{a}}$ & $7.3^{\mathrm{a}, \mathrm{b}}$ & $18.7^{\mathrm{a}, \mathrm{b}}$ & $14.3^{\mathrm{c}, \mathrm{d}}$ & $0.89^{\mathrm{a}, \mathrm{b}}$ & $7.1^{\mathrm{a}}$ & $15.4^{\mathrm{b}, \mathrm{c}}$ \\
Nycticorax nycticorax barba & $5.4^{\mathrm{a}}$ & $8.2^{\mathrm{a}, \mathrm{b}}$ & $7.2^{\mathrm{a}, \mathrm{b}}$ & $61.5^{\mathrm{a}, \mathrm{b}}$ & $21.3^{\mathrm{a}, \mathrm{b}, \mathrm{c}}$ & $0.34^{\mathrm{a}, \mathrm{b}}$ & $9.5^{\mathrm{a}}$ & $30.7^{\mathrm{b}}$ \\
N. nycticorax raquis & $5.4^{\mathrm{a}}$ & $9.3^{\mathrm{a}}$ & $7.3^{\mathrm{a}, \mathrm{b}}$ & $16.8^{\mathrm{b}}$ & $12.6^{\mathrm{c}, \mathrm{d}, \mathrm{e}}$ & $0.45^{\mathrm{a}, \mathrm{b}}$ & $8.6^{\mathrm{a}}$ & $12.0^{\mathrm{b}, \mathrm{c}}$ \\
Agua del lago & $0.01^{\mathrm{c}}$ & $0.02^{\mathrm{c}}$ & $0.02^{\mathrm{b}}$ & $0.02^{\mathrm{b}}$ & $0.01^{\mathrm{e}}$ & $0.01^{\mathrm{b}}$ & $0.02^{\mathrm{b}}$ & $0.03^{\mathrm{c}}$ \\
Sedimento & $0.4^{\mathrm{c}}$ & $0.4^{\mathrm{c}}$ & $0.6^{\mathrm{b}}$ & $4.9^{\mathrm{b}}$ & $2.6^{\mathrm{d}, \mathrm{e}}$ & $0.5^{\mathrm{a}, \mathrm{b}}$ & $1.4^{\mathrm{b}}$ & $118.8^{\mathrm{a}}$ \\
\hline
\end{tabular}

Valores con la misma letra en la columna son estadísticamente iguales $(\mathrm{p} \leq 0.05)$ 


\section{Cobre}

La especie que presentó la mayor concentración en las barbas de plumas fue E. thula, con $34.57 \mu \mathrm{g} / \mathrm{g}$, seguida por $A$. alba con $27.78 \mu \mathrm{g} / \mathrm{g}$ y $N$. nycticorax con $21.3 \mu \mathrm{g} / \mathrm{g}$. A. alba obtuvo la concentración más alta de $\mathrm{Cu}$ en el raquis con $20.5 \mu \mathrm{g} / \mathrm{g}$, seguida por $E$. thula con $14.3 \mu \mathrm{g} / \mathrm{g}$ y $N$. nycticorax con $12.56 \mu \mathrm{g} / \mathrm{g}$. No se encontró diferencia significativa entre las tres especies. El $\mathrm{Cu}$ es un metal esencial, sin embargo se ha podido observar que en altas concentraciones induce daños en aves como anemia, trastornos del crecimiento, respiratorios, carcinogénicos, reproductivos, gastrointestinales, hematológicos, hepáticos y endocrinos (Skoric et al. 2012, Manjula et al. 2015).

\section{Plomo}

$\mathrm{El} \mathrm{Pb}$ es un metal no esencial con alta toxicidad de importancia para la salud humana y ambiental; tiende a interactuar con el calcio, por lo cual se encuentra principalmente en plumas y huesos de aves (EstradaGuerrero y Soler-Tovar 2015). No hubo diferencias significativas entre las concentraciones encontradas en las distintas especies. Padula et al. (2010) obtuvieron concentraciones de $2.27,1.35$ y $1.76 \mu \mathrm{g} / \mathrm{g}$ en $N$. nycticorax en tres distintas colonias de una misma zona. Por su parte Custer et al. (2008) encontraron en esta misma ave concentraciones de $0.32,0.11$, y $0.41 \mu \mathrm{g} / \mathrm{g}$ en tres diferentes colonias, las cuales están por debajo de las reportadas en este estudio (9.5 y $8.5 \mu \mathrm{g} / \mathrm{g})$, Abdullah et al. (2015) obtuvieron concentraciones de $\mathrm{Pb}$ en B. ibis de 297 y $286 \mu \mathrm{g} / \mathrm{g}$ en dos diferentes localidades, por arriba de las encontradas en las especies de este estudio. Cardiel et al. (2011) establecieron que una concentración de $1.5 \mu \mathrm{g} / \mathrm{g}$ de $\mathrm{Pb}$ en el raquis de las plumas, constituye una exposición anormal. Por otro lado, Tsipoura et al. (2011) sostienen que concentraciones a partir de $4 \mathrm{mg} / \mathrm{kg}$ de $\mathrm{Pb}$ en plumas están asociadas con comportamiento, termorregulación, locomoción y percepción profunda, y resultan en menor supervivencia de los pichones, además de anemia hemolítica (Lucia et al. 2010), daños gastrointestinales y neurológicos que se reflejan en el comportamiento de las aves, y finalmente la muerte (Estrada-Guerrero y Soler-Tovar 2014). Las concentraciones de $\mathrm{Pb}$ encontradas en este estudio sobrepasan de 0.5 a 2.1 veces las reportadas en dicho estudio, lo cual indica la presencia de contaminación que puede estar causando efectos adversos.

\section{Arsénico}

Las concentraciones de As en las especies de garzas del lago, no presentaron diferencias significativas entre la barba o el raquis de las plumas. Padula et al.
(2010) reportaron concentraciones de $0.213 \mu \mathrm{g} / \mathrm{g}$ en $N$. nycticorax en la isla Goose, $0.467 \mu \mathrm{g} / \mathrm{g}$ en la isla North Brother, y $0.114 \mu \mathrm{g} / \mathrm{g}$ en la isla Hoffman (Nueva York). Estos valores son hasta 20 veces menores que las concentraciones obtenidas en las tres especies del lago de Chapala. En aves, el As puede causar trastornos reproductivos, hematológicos, hepático genéticos, cambios en el comportamiento y problemas inmunológicos (Sánchez-Virosta et al. 2015).

\section{Cadmio}

En este estudio no se observaron diferencias significativas entre las concentraciones de $\mathrm{Cd}$ en las tres garzas del lago. En Pakistán, Abbasi et al. (2015) encontraron concentraciones de $1.03 \mu \mathrm{g} / \mathrm{g}$ de Cd en N. nycticorax. En India, Manjula et al. (2015) reportaron concentraciones de Cd en Egreta garzzeta de 1.55 y $1.54 \mu \mathrm{g} / \mathrm{g}$ en dos localidades distintas. Las concentraciones reportadas en las especies de garzas del Lago de Chapala son hasta 4.7 veces superiores que las reportadas por estos autores. Se ha informado que en aves este metal es tóxico en concentraciones mayores a $3 \mu \mathrm{g} / \mathrm{g}$, e incluso en concentraciones más bajas $(0.1-2 \mu \mathrm{g} / \mathrm{g})$ puede alterar los patrones de crecimiento y causar efectos letales (Burger 2013).

\section{Plata}

No se encontraron diferencias significativas en las concentraciones de $\mathrm{Ag}$, en la barba y raquis de las distintas especies de garzas del lago. La fuente de contaminación por Ag es de tipo industrial y las sales de Ag son tóxicas para peces de agua dulce, pero aún se desconocen los efectos producidos en aves acuáticas (Förstner 1981).

\section{Mercurio}

En los ecosistemas acuáticos, el mercurio puede provenir de la precipitación atmosférica o de la descarga de aguas residuales, principalmente industriales. Una vez que ingresa en los sistemas acuáticos, este elemento puede permanecer en el agua o depositarse en los sedimentos (Herruzo et al. 2010). Se encuentra en regiones donde se utilizan herbicidas, fungicidas y conservadores de semillas, y donde hay presencia de industrias como las de pinturas, papeleras y electroquímicas (Villarejo 2004). En aves, el $\mathrm{Hg}$ es un potente neurotóxico, lo cual pone de manifiesto el riesgo de este metal en el ecosistema (Aggarwal et al. 2014). No se observan diferencias significativas para este metal tanto en raquis como en barba de todas las especies de aves estudiadas. La barba de E. thula tiene una concentración 0.8 a 0.3 veces mayor que el resto de las aves. Sin embargo, 
en años anteriores Torres et al. (2014) encontraron tres a cuatro veces más $\mathrm{Hg}$ que el reportado en este estudio en aves migratorias del mismo lago como $P$. erythrorhynchos e inclusive en $A$. alba, lo cual indica que la contaminación varía de estación a estación y de año en año.

\section{Bioacumulación}

La acumulación de metales pesados en plumas de aves se determinó con el FBSB. Los resultados se muestran en el Cuadro II. Un número mayor a uno indica bioacumulación. Ésta se presentó en las tres especies de aves acuáticas y con la mayoría de los metales, a excepción del Ti.

Para Ag, As y Cd no se observan diferencias significativas en el raquis ni en la barba de las tres especies. Sin embargo, el Cr se acumula más en la barba de $A$. alba respecto al resto de las aves. La bioacumulación de $\mathrm{Cu}$ mostró una diferencia significativa $(\mathrm{p}=0.0012)$ en la barba de $E$. thula respecto al raquis de E. thula y $N$. nycticorax. Para el $\mathrm{Hg}$ tanto el raquis como la barba de las tres especies fueron diferentes significativamente $(\mathrm{p}=0.001)$ con excepción del raquis de $A$. alba y la barba de $N$. Nycticorax. Por lo tanto, en este estudio $E$. thula bioacumuló más metales pesados que el resto de las especies.

Para el $\mathrm{Pb}$ se observa una diferencia significativa $(\mathrm{p}=0.0028)$ entre el raquis de $A$. alba y el resto de las muestras, lo que indica que existe mayor bioacumulación en el raquis de esa ave. Respecto al Ti, a pesar de existir diferencias significativas entre las muestras $(\mathrm{p}=0.0015)$ no se observó acumulación de este metal ni en raquis ni en barba de ninguna especie.

Estos resultados indican que hubo bioacumulación de metales pesados del sedimento en las plumas de las aves acuáticas estudiadas del lago de Chapala. Cabe resaltar que la bioacumulación de metales es variable entre las especies de aves incluidas en el estudio. Las plumas son buenas indicadoras de contaminación por metales debido a que en ellas se acumulan los contaminantes que no utilizan para su metabolismo (Luoma y Rainbow 2005).

El FBSB se ha utilizado en distintas partes del mundo para medir la bioacumulación de sedimentobiota de distintos contaminantes como los policlorobifenilos, compuestos organoclorados y metales pesados (principalmente $\mathrm{Hg}$ ) (Beek et al. 2000, Calle et al. 2015, Lui et al. 2016). Ruelas-Inzunza et al. (2011b) utilizaron el FBSB para medir la bioacumulación de $\mathrm{Cd}, \mathrm{Cr}, \mathrm{Hg}$ y $\mathrm{Pb}$ en 12 especies de peces y 11 especies de crustáceos en un área asociada a la minería en la cuenca del río Baluarte, en la parte noroeste de México. Dichos autores encontraron bioacumulación de $\mathrm{Hg}$ en los peces (FBSB $>1$ ), por lo que es probable que las aves, que son piscívoras, acumulen los contaminantes por ingesta. Además, hay otras vías, como el contacto con el agua del lago. Así, el uso de este factor es relevante, debido a que no sólo se enfoca a los contaminantes que ingieren por la dieta los organismos estudiados, sino por la contaminación presente en el agua y en los sedimentos de este humedal. El siguiente paso de este tipo de investigaciones será abordar los efectos que dicha contaminación tiene en las aves acuáticas y su posible implicación en la salud humana.

\section{CONCLUSIÓN}

Las aves acuáticas residentes del lago de Chapala, A. alba, E. thula y N. nycticorax, demostraron ser buenos indicadores de la contaminación por metales pesados, los cuales se encontraron en concentraciones altas en las plumas de las tres especies. El uso de plumas de aves para el biomonitoreo de metales pesados es una excelente herramienta, ya que constituye una forma no invasiva ni letal de medir la concentración de metales. Los valores del FBSB de los diferentes metales pesados confirman que la biota del lago de Chapala presenta una acumulación importante de

CUADRO II. FACTORES DE ACUMULACIÓN DE SEDIMENTO-BIOTA DE TRES ESPECIES DE AVES RESIDENTES DEL LAGO DE CHAPALA

\begin{tabular}{|c|c|c|c|c|c|c|c|c|}
\hline Matriz & $\mathrm{Ag}$ & As & $\mathrm{Cd}$ & $\mathrm{Cr}$ & $\mathrm{Cu}$ & $\mathrm{Hg}$ & $\mathrm{Pb}$ & $\mathrm{Ti}$ \\
\hline Ardea alba raquis & $13.3^{\mathrm{a}}$ & $20.1^{\mathrm{a}}$ & $11.8^{\mathrm{a}}$ & $10.8^{\mathrm{a}, \mathrm{b}}$ & $12.4^{\mathrm{a}, \mathrm{b}}$ & $0.72^{\mathrm{e}}$ & $31.4^{\mathrm{a}}$ & $0.09^{\mathrm{c}, \mathrm{b}}$ \\
\hline A. Alba barba & $10.4^{\mathrm{a}, \mathrm{b}}$ & $14.5^{\mathrm{b}}$ & $9.3^{\mathrm{a}}$ & $23.8^{\mathrm{a}}$ & $10.8^{\mathrm{a}, \mathrm{b}}$ & $1.24^{\mathrm{c}}$ & $5.1^{\mathrm{b}}$ & $0.09^{\mathrm{b}, \mathrm{c}}$ \\
\hline Egretta. thula barba & $13.3^{\mathrm{a}}$ & $18.9^{\mathrm{a}}$ & $11.7^{\mathrm{a}}$ & $12.1^{\mathrm{a}, \mathrm{b}}$ & $13.4^{\mathrm{a}}$ & $2.22^{\mathrm{a}}$ & $6.4^{\mathrm{b}}$ & $0.3^{\mathrm{a}}$ \\
\hline E. thula raquis & $13.3^{\mathrm{a}}$ & $23.2^{\mathrm{a}}$ & $11.9^{\mathrm{a}}$ & $3.8^{\mathrm{b}}$ & $5.5^{\mathrm{b}, \mathrm{c}}$ & $1.78^{\mathrm{b}}$ & $5.1^{\mathrm{b}}$ & $0.12^{\mathrm{b}}$ \\
\hline Nycticorax nycticorax barba & $13.3^{\mathrm{a}}$ & $20.1^{\mathrm{a}}$ & $11.8^{\mathrm{a}}$ & $17.2^{\mathrm{b}}$ & $8.3^{\mathrm{a}, \mathrm{b}, \mathrm{c}}$ & $0.68^{\mathrm{e}}$ & $6.8^{\mathrm{b}}$ & $0.3^{\mathrm{a}}$ \\
\hline N. nycticorax raquis & $13.3^{\mathrm{a}}$ & $23.0^{\mathrm{a}}$ & $11.8^{\mathrm{a}}$ & $3.5^{\mathrm{a}, \mathrm{b}}$ & $4.9^{\mathrm{b}, \mathrm{c}}$ & $0.9^{\mathrm{d}}$ & $6.2^{\mathrm{b}}$ & $0.10^{\mathrm{b}, \mathrm{c}}$ \\
\hline
\end{tabular}

Valores con la misma letra en la columna son estadísticamente iguales $(\mathrm{p} \leq 0.05)$ 
éstos en relación con concentraciones encontradas en el sedimento. Lo anterior es un paso importante hacia la valoración de la salud del ecosistema y los posibles riesgos para sus habitantes. Los resultados de este trabajo son una base para futuros estudios que valoren los efectos o impactos de los metales en aves residentes de humedales como el lago de Chapala.

\section{AGRADECIMIENTOS}

A la Secretaría de Investigación y Posgrado del Instituto Politécnico Nacional por el financiamiento (SIP 20140330 y 20150328), al Dr. T. Silva y el Dr. G. Cruz por su ayuda en el muestreo de agua y sedimentos, y a Uriel Lorenzo García por la elaboración del mapa de localización. D. González agradece al CONACyT por la beca otorgada para realizar sus estudios de maestría.

\section{REFERENCIAS}

Abbasi N.A., Jaspers V.L.B., Chaudhry M.J.I., Ali S. y Malik R.N. (2015). Influence of taxa, trophic level, and location on bioaccumulation of toxic metals in bird's feathers: A preliminary biomonitoring study using multiple bird species from Pakistan. Chemosphere 120, 527-537. DOI: 10.1016/j.chemosphere.2014.08.054

Abdullah M., Fasola M., Muhammad A., Malik S.A., Bostan N., Bokhari H. y Eqani S.A. (2015). Avian feathers as a non-destructive bio-monitoring tool of trace metals signatures: A case study from severely contaminated areas. Chemosphere 119, 553-561.

DOI: 10.1016/j.chemosphere.2014.06.068

Aggarwal P., Gaur S. y Gauba P. (2014). Neurotoxic and genotoxic effects of methylmercury. Environ. Dev. Sustain. 16 (1), 71-78.

DOI: $10.1007 / \mathrm{s} 10668-013-9475-8$

Beek B., Böhling S., Bruckmann U., Franke C., Jöhncke U. y Studinger G. (2000). The assessment of bioaccumulation. En: Bioaccumulation-New aspects and developments (B. Beek, Ed.) Springer Berlin Heidelberg, Springer Science and Business Media, Berlín, Alemania, pp. 235-276.

DOI: $10.1007 / 10503050$

Brito E.M., de la Cruz Barrón M., Caretta C.A., GoñiUrriza M., Andrade L.H., Cuevas-Rodríguez G. y Guyoneaud R. (2015). Impact of hydrocarbons, PCBs and heavy metals on bacterial communities in Lerma River, Salamanca, Mexico: Investigation of hydrocarbon degradation potential. Sci. Total Environ. 521, 1-10. DOI: 10.1016/j.scitotenv.2015.02.098
Burger J. y Gochfeld M. (2004). Marine birds as sentinels of environmental pollution. EcoHealth 1 (3), 263-274. DOI: 10.1007/s10393-004-0096-4

Burger J. Gochfeld M., Sullivan K., Irons D. y McKnight A. (2008). Arsenic, cadmium, chromium, lead, manganese, mercury, and selenium in feathers of Black-legged Kittiwake (Rissa tridactyla) and Black Oystercatcher (Haematopus bachmani) from Prince William Sound, Alaska. Sci. Total Environ. 398 (1), 20-25. DOI: 10.1016/j.scitotenv.2008.02.051

Burger J. (2013). Temporal trends (1989-2011) in levels of mercury and other heavy metals in feathers of fledgling great egrets nesting in Barnegat Bay, NJ. Environ. Res. 122, 11-17. DOI: 10.1016/j.envres.2013.01.003

Calle P., Alvarado O., Monserrate L., Cevallos J.M., Calle N. y Alava J.J. (2015). Mercury accumulation in sediments and seabird feathers from the Antarctic Peninsula. Mar. Pollut. Bull. 91 (2), 410-417. DOI: 10.1016/j.marpolbul.2014.10.009

Cardiel I.E., Taggart M A. y Mateo R. (2011). Using Pb-Al ratios to discriminate between internal and external deposition of $\mathrm{Pb}$ in feathers. Ecotox. Environ. Safe. 74 (4), 911-917.

DOI: 10.1016/j.ecoenv.2010.12.015

Custer T.W., Golden N.H. y Rattner B.A. (2008). Element patterns in feathers of nestling black-crowned nightherons, Nycticorax nycticorax L., from four colonies in Delaware, Maryland, and Minnesota. B. Environ. Contam. Tox. 81 (12), 147-151. DOI: $10.1007 / \mathrm{s} 00128-008-9444-3$

Dauwe T., Bervoets L., Pinxten R., Blust R. y Eens M. (2003). Variation of heavy metals within and among feathers of birds of prey: Effects of molt and external contamination. Environ. Pollut. 124 (3), 429-436. DOI: 10.1016/S0269-7491(03)00044-7

Estrada-Guerrero D.M. y Soler-Tovar D. (2014). Las aves como bioindicadores de contaminación por metales pesados en humedales. Ornitología Colombiana 14, 145-160.

Ferreira A.P. (2011). Assessment of heavy metals in Egretta thula: Case study: Coroa Grande mangrove, Sepetiba Bay, Rio de Janeiro, Brazil. Braz. J. Biol. 71 (1), 77-82. DOI: 10.1590/S1519-69842011000100012

Förstner U. (1981). Metal transfer between solid and aqueous phases. En: Metal pollution in the aquatic environment (Förstner U. y Wittmann G.T., Eds.). Springer Science \& Business Media. Berlín, Alemania, p. 502.

García-Fernández A.J., Espín S. y Martínez-López E. (2013). Feathers as a biomonitoring tool of polyhalogenated compounds: A review. Environ. Sci. Technol. 47 (7), 3028-3043. DOI: 10.1021/es302758x

Gutiérrez R., Barba G. y del Toro M.R. (2008). Ficha Informativa de los Humedales de Ramsar (FIR), 
versión 2006-2008 [en línea]. http://semadet.jalisco. gob.mx/sites/semadet.jalisco.gob.mx/files/fichalagodechapala.pdf

Hansen A.M. y van Afferden M. (2004). El Lago de Chapala: destino final del río Lerma. En: El agua en México vista desde la academia (B. Jiménez y L. Marín, Eds.). Academia Mexicana de Ciencias, Ciudad de México, México, pp. 117-135.

Honda K., Min B.Y. y Tatsukawa R. (1986). Distribution of heavy metals and their age-related changes in the eastern great white egret, Egretta alba modesta, in Korea. Arch. Environ. Contam. Tox. 15 (2), 185-197. DOI: 10.1007/BF01059967

Herruzo F.G., Rubio A.G., Lahoz C.G., Alonso C.V. y Maroto J.M.R. (2010). El mercurio: situación actual, problemas y soluciones. Ingeniería Química 480, 84-91.

INEGI (2016). Mapas. Instituto Nacional de Estadística, Geografía e Informática [en línea]. http://www.beta. inegi.org.mx/temas/mapas/climatologia/ 20/07/2015.

Lodenius M. y Solonen T. (2013). The use of feathers of birds of prey as indicators of metal pollution. Ecotoxicology 22 (9), 1319-1334.

DOI: $10.1007 / \mathrm{s} 10646-013-1128-\mathrm{z}$

Lucia M., André J.M., Gontier K., Diot N., Veiga J. y Davail S. (2010). Trace element concentrations (mercury, cadmium, copper, zinc, lead, aluminium, nickel, arsenic, and selenium) in some aquatic birds of the Southwest Atlantic Coast of France. Arch. Environ. Contam. Toxicol. 58(3), 844-853.

DOI: $10.1007 / \mathrm{s} 00244-009-9393-9$

Luoma S.N. y Rainbow P.S. (2005). Why is metal bioaccumulation so variable? Biodynamics as a unifying concept. Environ. Sci. Technol. 39 (7), 1921-1931. DOI: $10.1021 / \mathrm{es} 048947 \mathrm{e}$

Manjula M., Mohanraj R. y Devi M.P. (2015). Biomonitoring of heavy metals in feathers of eleven common bird species in urban and rural environments of Tiruchirappalli, India. Environ. Monit. Assess. 187 (5), 1-10.

DOI: $10.1007 / \mathrm{s} 10661-015-4502-\mathrm{x}$

Moncayo-Estrada R. y Buelna-Osben H.R. (2001). Fish fauna of Lake Chapala. In The Lerma-Chapala Watershed. (A. M. Hansen and M. van Afferden, Eds.). Springer US. Nueva York, EUA, pp. 215-242.

Misztal-Szkudlińska M., Szefer P., Konieczka P. y Namieśnik J. (2012). Mercury in different feather types from great cormorants (Phalacrocorax carbo L.) inhabiting the vistula lagoon ecosystem in Poland. B. Environ. Contam. Tox. 89 (4), 841-844.

DOI: $10.1007 / \mathrm{s} 00128-012-0771-\mathrm{z}$

Nam D.H., Anan Y., Ikemoto T., Okabe Y., Kim E.Y., Subramanian A. y Tanabe S. (2005). Specific accumulation of 20 trace elements in great cormorants
(Phalacrocorax carbo) from Japan. Environ. Pollut. 134 (3), 503-514. DOI: 10.1016/j.envpol.2004.09.003

Parra O.E. (2014). Aves silvestres como bioindicadores de contaminación ambiental y metales pesados. Revista CES Salud Pública 5 (1), 59-69.

Padula V., Burger J., Newman S.H., Elbin S. y Jeitner C. (2010). Metals in feathers of black-crowned nightheron (Nycticorax nycticorax) chicks from the New York Harbor Estuary. Arch. Environ. Contam. Tox. 59 (1), 157-165. DOI: 10.1007/s00244-009-9427-3

Rosales-Hoz L., Carranza-Edwards A. y López-Hernández M. (2000). Heavy metals in sediments of a large, turbid tropical lake affected by anthropogenic discharges. Environ. Geol. 39 (3), 378-383.

DOI: $10.1007 / \mathrm{s} 002540050017$

Ruelas-Inzunza J., Hernández-Osuna J. y Páez-Osuna F. y Zamora-Arellano N. (2009). Organic and total mercury in muscle tissue of five aquatic birds with different feeding habits from the SE Gulf of California, Mexico. Chemosphere 76 (3), 415-418.

DOI: 10.1016/j.chemosphere.2009.03.042

Ruelas-Inzunza J., Páez-Osuna F. y Ruiz-Fernández A.C. (2011a). Health Risk Associated to Dietary Intake of Mercury in Selected Coastal Areas of Mexico. B. Environ. Contam. Tox. 86 (2), 180.

DOI: $10.1007 / \mathrm{s} 00128-011-0189-\mathrm{z}$

Ruelas-Inzunza J., Green-Ruiz C., Zavala-Nevárez M. y Soto-Jiménez M. (2011b). Biomonitoring of Cd, Cr, $\mathrm{Hg}$ and $\mathrm{Pb}$ in the Baluarte River basin associated to a mining area (NW Mexico). Sci. Total Enviro. 409 (18), 3527-3536. DOI: 10.1016/j.scitotenv.2011.05.035

Sánchez-Virosta P., Espín S., García-Fernández A.J. y Eeva T. (2015). A review on exposure and effects of arsenic in passerine birds. Sci. Total Environ. 512-513, 506-525.

DOI: 10.1016/j.scitotenv.2015.01.069

SAS (2004). SAS Institute Inc. SAS/STAT ${ }^{\circledR} 9.1$ user's guide. SAS Institute Inc., Cary, NC, USA, 5136 pp.

Sedeño-Díaz J.E. y López-López E. (2007). Water quality in the río Lerma, Mexico: An overview of the last quarter of the twentieth century. Water Resour. Manag. 21 (10), 1797-1812. DOI: 10.1007/s11269-006-9128-x

Sfakianakis D.G., Renieri E., Kentouri M. y Tsatsakis A.M. (2015). Effect of heavy metals on fish larvae deformities: A review. Environ. Res. 137, 246-255. DOI: 10.1016/j.envres.2014.12.014

Skoric S., Visnjić-Jeftic Z., Jaric I., Djikanovic V., Mickovic B., Nikcevic M. y Lenhardt M. (2012). Accumulation of 20 elements in great cormorant (Phalacrocorax carbo) and its main prey, common carp (Cyprinus carpio) and Prussian carp (Carassius gibelio). Ecotox. Environ. Safe. 80, 244-25.

DOI: 10.1016/j.ecoenv.2012.03.004 
Torres Z., Mora M.A., Taylor R.J., Álvarez-Bernal D., Buelna H.R. y Hyodo A. (2014). Accumulation and hazard assessment of mercury to waterbirds at Lake Chapala, Mexico. Environ. Sci. Technol. 48 (11), 63596365. DOI: $10.1021 / \mathrm{es} 4048076$

Torres Z., Mora M.A., Taylor R.J. y Álvarez-Bernal D. (2016). Tracking metal pollution in Lake Chapala: Concentrations in water, sediments, and fish. B. Environ. Contam. Tox. 97 (3), 418-424. DOI: $10.1007 / \mathrm{s} 00128-016-1892-6$

Trujillo-Cárdenas J.L., Saucedo-Torres N.P., Zárate del Valle P.F., Ríos-Donato N., Mendizábal E. y GómezSalazar S. (2010). Speciation and sources of toxic metals in sediments of Lake Chapala, Mexico. J. Mex. Chem. Soc. 54 (2), 79-87.
Tsipoura N., Burger J., Newhouse M., Jeitner C., Gochfeld M. y Mizrahi D. (2011). Lead, mercury, cadmium, chromium, and arsenic levels in eggs, feathers, and tissues of Canada geese of the New Jersey Meadowlands. Environ. Res. 111 (6), 775-784. DOI: 10.1016/j.envres.2011.05.013

Villarejo A.L D. (2004). Ecotoxicología y acción toxicológica del mercurio. Anal. Real Acad. Nac. Farm. 70 (4), 933-959.

Zhang W. y Ma J.Z. (2011). Waterbirds as bioindicators of wetland heavy metal pollution. Procedia Environ. Sci. 10, 2769-2774. DOI: 10.1016/j.proenv.2011.09.429 